die ökonomischen Instrumente an, dass Emissionsvermeidungs- und Umweltverbesserungsmaßnahmen vor allem dort ausgelöst werden, wo sie verhältnismäßig kostengünstig sind. Aufgrund der Flexibilität für die Betroffenen, die sich jeweils für die Zahlung einer Abgabe bzw. den Kauf eines Zertifikates oder aber für Umweltweltschutzmaßnahmen entscheiden können, gelten die ökonomischen Instrumente zudem als relativ freiheitsfreundlich.

Grundsätzlich wäre denkbar, Lebensmittelabfälle direkt zu verteuern, etwa durch einen hohen Steuersatz. Mit Blick auf die für die Lebensmittelabfälle bedeutsamen Haushaltsabfälle - aber auch bezogen auf weitere Lebensmittelabfälle entlang der PLK (Kapitel 1.2) - wurde indes deutlich, dass diese schwer administrativ zu erfassen und zu kontrollieren sind. Schon eher denkbar ist eine umfassendere DeponieSteuer, wie sie beispielsweise die Niederlande im Jahr 2015 (wieder-)eingeführt haben. Die Höhe der Steuer liegt bei 17 Euro pro Tonne. Gleichzeitig wurde die Deponierung von kommunalen und recycling-fähigen Abfällen verboten. ${ }^{156}$ Da diese Maßnahmen nicht explizit Lebensmittelabfälle betreffen, müssen diese für die Steuererhebung nicht genau quantifiziert werden. Ob eine derartige Steuer Lebensmittelabfälle umfassend entlang der gesamten PLK entgegenwirken könnte, erscheint jedoch zweifelhaft, zumal insbesondere das Recycling der Abfälle gefördert wird, die Vermeidung aber nicht zwingend priorisiert wird.

Einen Beitrag zur Reduktion der Lebensmittelabfälle, insbesondere jener auf Haushaltsebene, würden vor allem höhere Preise für Lebensmittel leisten. ${ }^{157}$ Andernorts wurde näher aufgezeigt, dass sich - weil weit größere Effekte als allein Umbauten etwa am System der Agrarsubventionen auslösend - zur integrierten Lösung verschiedener Umweltprobleme ein europäisches Primärenergie-Emissionshandelssystem (im Cap an Art. 2 Abs. 1 PA orientiert) in Kombination mit einem Emissionshandel für tierische Produkte eignet. ${ }^{158}$ Durch stark steigende Preise besonders für tierische Produkte würde nicht nur ein Anreiz gesetzt, diese weniger zu konsumieren und anders $\mathrm{zu}$ produzieren, sondern auch Lebensmittelabfälle in diesem besonders ressourcenintensiven und umweltschädlichen Bereich zu vermeiden. Dadurch, dass fossile Brennstoffe in nahezu allen Lebens- und Wirtschaftsbereichen einschließlich der Landwirtschaft eingesetzt werden, würde ein strenges Cap, welches die fossilen Brennstoffe innerhalb von nicht mehr als zwei Dekaden aus dem Markt nimmt, nicht zuletzt auch zu höheren Produktionskosten im Agrarsektor und damit $\mathrm{zu}$ steigenden Lebensmittelpreisen führen, wodurch neben Effizienz und Konsistenz auch Suffizienz - einschließlich der Vermeidung von Lebensmittelabfällen - angereizt würde. ${ }^{159}$ Kombiniert werden müsste ein solcher EU-Ansatz mit Border Adjustments gegenüber Ländern ohne ähnlichen Steuerungsansatz. Letzteres steht im Rahmen des European Green Deal aktuell denn auch auf der EU-Tagesordnung.

156) Vgl. Aramyan/Valeva, FUSIONS, S. 7.

157) Vgl. neben den Nachweisen aus Fn. 155 auch Danish Council on Ethics, The Ethical Consumer. Climate Damaging Foods, 2016, S. 11; BMEL, Grünbuch Ernährung, Landwirtschaft, Ländliche Räume. Gute Ernährung, starke Landwirtschaft, lebendige Regionen, 2016, S. 15

158) Ergänzt zudem um eine Flächenbindung - näher vgl. Weishaupt, Nachhaltigkeits-Governance; Ekardt, Sustainability, Ch. 4.6, Hennig, Nachhaltige Landnutzung und Bioenergie. Ambivalenzen, Governance, Rechtsfragen, 2017; Garske, Instrumente, Kapitel 5 .

159) Ausführlich dazu Ekardt, Sustainability, Ch. 4.6. Siehe auch IPCC, Global Warming of $1.5^{\circ} \mathrm{C}, 2018$; Rockström u. a., Science 2017; Ekardt/Wieding/Zorn, Sustainability 2018; Steffen u.a., PNAS 2018.

TAGUNGSBERICHTE

\title{
https://doi.org/10.1007/s10357-021-3815-5
}

\section{Grüner Wasserstoff im Recht}

\author{
Bericht zur Tagung des Vereins für Infrastrukturrecht (VIR) in Kooperation \\ mit der "Initiative on Energy and Policy“ der Bucerius Law School vom 5. November 2020

\section{Caterina Freytag/Kaspar Herbst}

Die vom Verein für Infrastrukturrecht (VIR) in Kooperation mit der „Initiative on Energy Law and Policy“ der Bucerius Law School ausgerichtete Tagung „Grüner Wasserstoff im Recht" nahm die nationale Wasserstoffstrategie (NWS) der Bundesregierung zum Anlass, um am 5.11.2020 über Rechtsfragen und Herausforderungen zu diskutieren, die mit dem Hochlauf einer marktfähigen Wasserstoffwirtschaft einhergehen. Die hybrid ausgerichtete Veranstaltung stieß mit über 150 Teilnehmer auf reges Interesse und be-

Caterina Freytag, Wissenschaftliche Mitarbeiterin,

Kaspar Herbst, Wissenschaftlicher Mitarbeiter,

Forschungsstelle für Europäisches Umweltrecht,

Universität Bremen,

Bremen, Deutschland wies damit nicht nur ein gutes Gespür für den drängenden Klärungs- und Austauschbedarfs, sondern bot diesem - mit Unterstïtzung der Bremer Kanzlei BBG und Partner - unter Pandemiebedingungen einen angemessenen Rahmen.

Nach einem Grußwort durch Peter Schütte (BBG und Partner) richtete Ingbert Liebing (VKU) in seiner Keynote das Augenmerk auf kommunale Chancen und Anforderungen im Zuge der NWS. Ein Fördervolumen von 9 Mrd. Euro sowie 38 Einzelmaßnahmen, die eine Leistungssteigerung der Wasserstoffproduktionsanlagen auf insgesamt $10 \mathrm{GW}$ bis 2040 anstreben, zeuge von der großen Bedeutung, die dem Wasserstoff von der Bundesregierung beigemessen werde. Da der Wärmemarkt auch nach Ausschöpfen von Effizienzund Elektrifizierungspotentialen voraussichtlich nicht vollständig auf gasförmige Energieträger werde verzichten können, komme grünem Wasserstoff eine Schlüsselrolle bei der 
angestrebten Dekarbonisierung zu (Stichwort: Power to X). Die erforderliche Infrastruktur müsse durch Umwidmung von Gasnetzen und entsprechende Governance-Strukturen jedoch frühzeitig angestoßen werden.

Wenn auch die erstmalige Schaffung eines konzeptionellen Rahmens durch die NWS zu begrüßen sei, gebe es aber auch erheblichen Nachbesserungsbedarf. Insbesondere seien, so Liebing, die Potentiale dezentraler Wasserstofferzeugung bzw. Verbrauchs vernachlässigt und zu stark auf industrielle Lösungen gesetzt worden. Außerdem bedürfe es einer Überprüfung des regulatorischen Rahmens. Diese sei zwar angekündigt worden, an konkreten Maßnahmen fehle es jedoch bislang.

\section{Rahmenbedingungen für Wasserstoffnutzung}

Matthias Schlegel (Fichtner GmbH \& Co. KG) ging zu Beginn des ersten Blocks der Frage nach, wie Wasserstoff in Deutschland zum Erfolg werden könne. Er legte nahe, verstärkt von der Anwendung her zu denken und insbesondere auch die Infrastruktur mit in den Blick zu nehmen. Zudem sei es förderlich, wenn Deutschland sich auf seine Rolle als Technologielieferant in diesem Bereich konzentriere.

Die anschließende Diskussion wurde auf das Gebot der Technologieneutralität hingewiesen und die Frage aufgeworfen, warum man die Entwicklung des Wasserstoffs nicht dem Markt überlassen könne. Diskussionsbedarf bestand auch mit Blick auf die infrastrukturellen Herausforderungen bei der Umstellung auf Wasserstoff, wobei angeregt wurde abschließend sich Japan zum Vorbild zu nehmen, das sich von Anfang an auf seine Rolle als Technologielieferant konzentriert habe.

Es folgte ein Vortrag von Christian Buchmüller (FH Westküste) zu den energie- und regulierungsrechtlichen Rahmenbedingungen der Wasserstoffwirtschaft. Bei der Erzeugung von Wasserstoff sah er das zentrale Problem in der Höhe der Gestehungskosten. Bei den staatlich induzierten Strompreisbestandteilen (SIP) sei die EEG-Umlage der große Posten. Hier kommen nach Ansicht Buchmüllers drei Privilegierungsmöglichkeiten in Betracht: Über das Speicherprivileg ( $\$ 61$ Abs. 1 EEG 2017), ein Eigenversorgungsprivileg (\$S 61a ff. EEG 2017) oder auch im Rahmen der besonderen Ausgleichsregelung (\$\$ 63f. EEG 2017). Jedoch seien alle Optionen mit gewissen Nachteilen behaftet. So setze das Speicherprivileg Rückverstromung voraus. Beim Eigenversorgungsprivileg seien immer noch $40 \%$ der EEG-Umlage $\mathrm{zu}$ entrichten und es gelten enge Voraussetzungen bei der Eigenversorgung. Auch die besondere Ausgleichsregelung biete keine Komplettbefreiung und könne nicht von allen Unternehmen in Anspruch genommen werden. Buchmüller plädierte hier mittelfristig für eine umfassende Reform der SIP inkl. EEG-Umlage. Kurzfristig sei die Einführung einer Komplettbefreiung von der EEG-Umlage für grünen Wasserstoff oder die umfassende Einbeziehung in die besondere Ausgleichsregelung wünschenswert. Hierbei müsste vor allem auf Investitionssicherheit und Konformität mit EU-Beihilferecht sowie Verfassungsrecht geachtet werden.

Hinsichtlich des Transports grünen Wasserstoffs ergeben sich nach Buchmüller zwei Möglichkeiten: die Beimischung von Wasserstoff in bestehende Netze und den Betrieb reiner Wasserstoffnetze. Gerade letzteres berge spannende Fragen zum grundsätzlichen Regulierungsbedarf, hinsichtlich der Zugangs- oder Entgeltregulierung und zu Finanzierungsmöglichkeiten. Für die Vermarktung des grünen Wasserstoffs fehle es bislang an spezifischen Anforderungen an die Vermarktung und den Vertrieb grünen Wasserstoffs. Hier bedürfe es in Umsetzung der RED II eines Herkunftsnachweissystems einschließlich der Definition von grünem Wasserstoff. Letzterer müsse auch mit bestehenden/neuen Fördergesetzen harmonisiert werden. Insgesamt fehle es hier noch an hinreichenden Anreizen und Privilegierungen für den Sektor.
In der sich anschließenden Diskussion wurde auf Rückfrage angedeutet, dass sich eine Präferenz für die besondere Ausgleichsregelung unter Ablehnung der Komplettbefreiungslösung abzeichne. Von Interesse waren zudem technische Umstellungsfragen der Gas- auf die Wasserstoffinfrastruktur.

\section{Möglichkeiten und Grenzen finanzieller Förderung}

Carsten Fichter (Hochschule Bremerhaven, ttz Bremen) gewährte zu Beginn des zweiten Themenblocks am Beispiel des seit 2018 laufenden Großforschungsprojekts „Grünes Gas für Bremerhaven“ Einblicke in standortbezogene Anwendungsfälle, darunter u.a. alternative Kraftstoffe (Stichwort: Methanisierung) aber auch Exoten wie den Wasserstoffofen. Fichter forderte dazu auf, die PtGWertschöpfungskette konsequent vom Ende her zu denken: Ohne verfügbare Anwendung keine Umwandlung. Zugleich mahnte er ein realistisches Erwartungsmanagement an: Eine mit hohen Wirkungsgradverlusten einhergehende Wasserstoffproduktion bedürfe, um wirtschaftlich $\mathrm{zu}$ sein, eines gezielten Einsatzes (jenseits bloßer Substitution von Erdgas im Wärmebereich). Auch auf grüne Überschussenergie, so räumte er auf Rückfrage ein, könne bei Umsetzung der EEG Novelle nicht länger gesetzt werden. Die Bundesregierung müsse absehbar die Ausbaumengen deutlich erhöhen oder auf Import von Wasserstoff setzen.

Martin Burgi (LMU München) widmete sich in der Frage der Wirtschaftlichkeit von auf grünem Wasserstoff basierender Technologie den Problemen der Beihilferechtskonformität. Angesichts des von der Bundesregierung forcierten Erhalts bzw. Ausbaus von Technologieführerschaft im Bereich Wasserstoff gilt das europäische Beihilferecht als besonders bedeutsam. Dieses greife unter Beachtung der hierfür jüngst vom EuGH aufgestellten Vorgaben, so Burgi, indes nicht im Falle der anvisierten Förderung über ein Einspeise- und Umlagesystems. Auch befürworte die Kommission augenscheinlich die geplante Förderung über sog. Carbon Contracts for Difference, wobei die Ausgestaltung im Einzelfall noch unklar und eine beihilferechtliche Prüfung deshalb gegenwärtig nicht möglich sei. Hoffnungsvoll stimmte Burgi in Bezug auf die derzeitig praktizierte Förderung mittels Investitionszuschuss: Komme eine Gruppenfreistellung gem. Art. 36, 40, 41, 48, 56 AGVO nicht in Betracht, folge die Freistellungsfähigkeit im Rahmen des Einzelnotifizierungsverfahrens grundsätzlich aus Art. 107 Abs. 3 lit. b) Var. 1 AEUV (Förderung wichtiger Vorhaben von gemeinsamen europäischen Interesse) bzw. Art. 107 Abs. 3 lit. c) Var. 1 AEUV (Förderung der Entwicklung gewisser Wirtschaftszweige) i.V.m. den Leitlinien für staatliche Umweltschutz- und Energiebeihilfen der Kommission. Wie anhand von Burgis Vortrag deutlich wurde, bestehen de lege lata keine unüberwindbaren beihilferechtlichen Hürden. Für mehr Rechtssicherheit und systemische Durchschlagkraft sei eine Weiterentwicklung des EU-Beihilfenrechts (etwa die Lückenschließung in den Ausnahmetatbeständen der AGVO) indes wünschenswert, um dem Wasserstoff eine normative Heimat zu geben. Ein gemeinsames Vorgehen auf Unionsebene sei unabdingbar (dies etwa in Bezug auf einheitliche Regelungen über Kennzeichen- und Nachweisanforderungen oder im Falle von Importen aus Drittstaaten bzw. Transport durch mehrere Mitgliedsstaaten) und die Umstände hierfür auch besonders günstig, da die Kommission ihrerseits auf Wasserstofftechnologie setze - wenn auch dem Grundsatz der Technikneutralität folgend nicht ausschließlich auf grünen Wasserstoff.

Die sich daran anschließende Diskussion hatte die Frage zum Gegenstand, ob eine Notifizierungspflicht ein Hindernis für eine gezielte Förderung erwünschter Technologien sei. Hingewiesen wurde insbesondere auf verwaltungspraktische Konsequenzen wie etwa die Vielzahl 
kleinteiliger Fördergeber, denen im Unterschied zur vorsorglichen Notifzierung des EEG 2017 durch das BMWi ein Notifizierungsverfahren nicht ohne Weiteres zuzumuten sei. Auch verzögere die Dauer des Notifizierungsverfahrens das Anlaufen der Förderprogramme erheblich. Im Verlauf der Diskussion wurde mit Blick auf die AltmarkTrans-Rechtsprechung des EuGH zudem angeregt, die Weiterentwicklung des EU-Beihilfenrechts nicht auf der Rechtfertigungsebene voranzutreiben, sondern durch teleologische Reduktion des Tatbestands. Burgi bezeichnete die Altmark-Trans-Rechtsprechung indes als Danaer Geschenk, da hier nur eine Verfahrenspflicht (Notizifierungsverfahren) durch eine andere (Vergabeverfahren) ersetzt worden sei.

\section{Planungs- und genehmigungsrechtlicher Rahmen}

Mit „eFarm“ und „Hyways for Future“ wurden zu Beginn des dritten Blocks zwei Pilotprojekte grüner Wasserstoffmobilität in Norddeutschland vorgestellt. Der Verkehrssektor gilt nach Auffassung der Referenten André Steinau (GP Joule) und Geert Tjarks (EWE AG) aufgrund seiner hohen Preisbereitschaft und Skalierbarkeit als primärer Anwendungsbereich grüner Wasserstofftechnologie - wenn die Flottenverfügbarkeit (im Vergleich zur Elektromobilität) auch noch ausbaufähig sei. Neben ihrer Bedeutung für die regionale Wertschöpfung seien diese Pilotprojekte heimische Keimzellen für Technologieentwicklung und -integration. Mit Blick auf die Speicherung von Wasserstoff habe, so Tjarks, (Nord)Deutschland zudem das Potential zum regenerativen Energiespeicher Europas zu werden. Insgesamt müsse der wasserstoffbasierte Infrastrukturausbau so auch stärker grenzübergreifend gedacht werden.

Sarah Langstädtler (BBG und Partner) untersuchte in ihrem Referat den genehmigungs- und planungsrechtlichen Rahmen für die Erzeugung, leitungsgebundene Verteilung sowie Speicherung von grünem Wasserstoff. Dabei arbeitete sie auf prägnante Weise heraus, welchen rechtlichen Unsicherheiten die Planung und Genehmigung von Elektrolyseuren bei der Frage des notwendigen Genehmigungsverfahrens und der UVP-Pflicht unterliegen. Zur Schaffung von Rechtssicherheit forderte Langstädtler Klarstellungen durch den Gesetz- bzw. Verordnungsgeber. Auch die geplante Errichtung eines Wasserstoff-Startnetzes sei, so ihre Analyse, genehmigungsrechtlich nicht abgesichert und mit der Situation der Gasversorgungsleitungen bis 2001 vergleichbar. Trotz des Umfangs eines Planfeststellungsverfahrens sei ein solches für die Errichtung neuer Wasserstofffernleitungen anzuraten, um die Vorhabenträger angesichts Investitionsvolumina im dreistelligen Millionenbereich in der Durchsetzung ihres Vorhabens rechtlich abzusichern. Bei der Speicherung grünen Wasserstoffs zeichnen nach Langstädlers Einschätzung hingegen (sowohl bei der über- als auch unterirdischen Speicherung) bislang keine besonderen planungs- oder genehmigungsrechtlichen Hürden ab.

In der Anschlussdiskussion wurde u. a. die planungsrechtliche Einordnung der angestrebten Umnutzung bestehender Gasversorgungsleitungen thematisiert. Auch wurde die Frage aufgeworfen, ob die vermeintliche Freiheit von rechtlichen Anforderungen für die Förderung von Wasserstoff tatsächlich der bessere Weg sei oder ob es hierzu nicht vielmehr eines dichten Rahmens (Stichwort: Technikermöglichungsrecht) in Gestalt eines eigenen Wasserstoffgesetzes bedürfe, wofür sich Langstädtler durchaus aufgeschlossen zeigte, sich zugleich aber gegen eine damit möglicherweise einhergehende Überregulierung verwahrte.

\section{Wasserstoff im Mobilitätssektor}

Die zwei abschließenden Projektsteckbriefe veranschaulichten die vielfältigen Einsatzmöglichkeiten des Wasser- stoffs in der Mobilitätswende. Während Georg Sandkühler (FAUN Gruppe) eine alternative Antriebstechnik für Nutzfahrzeuge (Müllentsorgungsfahrzeuge) mit Brennstoffzelle vorstellte, die ab 2021 in Serienproduktion gehen sollen, gewährte Thomas Nawrocki (LNVG) einen Ausblick auf den ab 2022 geplanten Einsatz von Wasserstoffzügen auf der Strecke Cuxhaven-Buxtehude, die als erste Schienenverbindung lokal emissionsfrei betrieben werden wird. Der hierfür benötigte Wasserstoff soll in Zukunft im Elektrolyseverfahren vor Ort aus grünem Strom gewonnen.

Zum Abschluss der Tagung verdeutlichte Matthias Knauff (Universität Jena) die vergaberechtlichen Anforderungen bei der Beschaffung von wasserstoffbetriebenen Fahrzeugen - dies auch gerade im Hinblick auf die Vorbildfunktion der öffentlichen Hand in Sachen Klimaschutz. Vorsicht sei geboten bei der Festlegung des Beschaffungsgegenstands und der Angebotswertung, wobei jedoch beides letztlich wasserstofffreundlich ausgestaltet werden könne. So seien bei der Festlegung des Beschaffungsgegenstands die Grenzen des Leistungsbestimmungsrechts des Auftraggebers zu beachten. Die Beschränkung auf (grün) wasserstoffbetriebene Fahrzeuge sei problematisch, wenn damit ein nicht gerechtfertigter Ausschluss anderer klimaverträglicher Antriebskonzepte einhergehe. Das Berücksichtigungsgebot von Umwelt- bzw. Klimaschutzaspekten gem. $₫ 68 \mathrm{VgV}$ (Stichwort: Emissionsfreiheit) helfe in diesem Zusammenhang allein nicht weiter. Mit Blick auf Technologieförderung seien etwa Innovationspartnerschaften als gesondertes Vergabeverfahren denkbar. Allerdings könne bereits durch die Hervorhebung von Einsatzzweck und Funktionalität eine hinreichende Konzentration auf wasserstoffbetriebene Antriebe erreicht werden, worauf auch in der anschließenden Diskussion hingewiesen wurde (etwa das kumulative Vorliegen von lokaler Emissionsfreiheit, Mindestnutzlast und Mindestreichweite). Auch in die Angebotswertung könne eine Präferenz für die Beschaffung von wasserstoffbetriebenen Fahrzeugen einfließen durch eine wasserstofffreundliche Ausgestaltung der Bewertungsmatrix. Als alleiniges Zuschlagskriterium sei der Wasserstoffantrieb jedoch sachlich nicht zu rechtfertigen. Insgesamt werde wasserstoffbetriebene Mobilität und die öffentliche Hand bei der Beschaffung wasserstoffbetriebener Fahrzeuge, sofern klug beraten, nicht am Vergaberecht scheitern, so Knauff.

\section{Zusammenfassung und Ausblick}

Das Fazit der Veranstalter, wie in den Schlussworten von Claudio Franzius (Bremen) und Michael Fehling (Hamburg) nach einer interdisziplinär wie hochkarätig besetzten $\mathrm{Ta}$ gung, die auch Praktiker und Pioniere zu Wort kommen ließ, deutlich wurde: Immer noch verwirrt, aber auf deutlich höherem Niveau. Man hätte abschließend auch die Losung des Referenten Fichter bemühen können: Nunmehr Einäugige unter Blinden.

Der Einsatz grünen Wasserstoffs verfügt über großes transformatives Potential, sein roll-out geht aber mit nicht minder großer Rechtsunsicherheit einher. Das Gelingen der Verkehrs- und Energiewende wird maßgeblich davon abhängen, ob die Weichen in diesem Bereich rechtzeitig und zielführend gestellt werden. Dies hat der VIR durch die Wahl und den Zuschnitt des Tagungsthemas mit dem Fokus auf grünen Wasserstoff erkannt und durch die Ausrichtung der Tagung einen wertvollen Beitrag hierzu geleistet. Viele Fragen wurden in diesem Zusammenhang aufgeworfen und bedürfen vertiefter Auseinandersetzung. Festzuhalten bleibt, dass grüner Wasserstoff zurecht als Hoffnungsträger gilt, dessen Markteinführung, wenn richtig aufgesetzt, jedenfalls nicht an vergabe- oder beihilferechtlichen Hürden scheitern wird. Es gilt aber, wie von Burgi angemerkt und im Verlauf der Tagung deutlich geworden, auch das regulatorische Umfeld für den benötigten Strom, für den künftigen Umgang mit $\mathrm{CO}_{2}$ und die übergreifenden As- 
pekte von Rechts-, Planungs- und damit auch Investitionssicherheit im Blick zu behalten. Grüner Wasserstoff wird sich am Markt nur in Verbindung mit einem konsequenten Ausbau erneuerbarer Energien und der hierfür notwendigen Infrastruktur bewähren können. Man darf in diesem Sinne auf die vom VIR im nächsten Jahr geplante Tagung $\mathrm{zu}$, ,Offshore-Infrastrukturen“ gespannt sein.

Open Access. Dieser Artikel wird unter der Creative Commons Namensnennung 4.0 International Lizenz veröffentlicht, welche die Nutzung, Vervielfältigung, Bearbeitung, Verbreitung und Wiedergabe in jeglichem Medium und Format erlaubt, sofern Sie den/die ursprünglichen Autor(en) und die Quelle ordnungsgemäß nennen, einen Link zur Creative Commons Lizenz beifügen und angeben, ob Änderungen vorgenommen wurden.

Die in diesem Artikel enthaltenen Bilder und sonstiges Drittmaterial unterliegen ebenfalls der genannten Creative Commons Lizenz, sofern sich aus der Abbildungslegende nichts anderes ergibt. Sofern das betreffende Material nicht unter der genannten Creative Commons Lizenz steht und die betreffende Handlung nicht nach gesetzlichen Vorschriften erlaubt ist, ist für die oben aufgeführten Weiterverwendungen des Materials die Einwilligung des jeweiligen Rechteinhabers einzuholen.

Weitere Details zur Lizenz entnehmen Sie bitte der Lizenzinformation auf http://creativecommons.org/licenses/by/4.0/deed.de.

Open Access funding enabled and organized by Projekt DEAL.

\title{
Schützt den Wald! - Das Verfahren „Skydda Skogen“ und seine artenschutzrechtlichen Folgen -
}

\author{
Anmerkung zu EuGH, Urteil vom 4.3.2021 - C-473/19 und C-474/19 (Skydda Skogen), NuR 2021, 186 \\ (in diesem Heft)
}

\section{Martin Gellermann und Jochen Schumacher}

(c) Springer-Verlag GmbH Deutschland, ein Teil von Springer Nature 2021.

Nachdem sich Generalanwältin Kokott in ihren Schlussanträgen in der Rechtssache „Skydda Skogen“ (zu Deutsch: Schützt den Wald) für eine Interpretation des Absichtsbegriffs des Art. 5 der Vogelschutz-Richtlinie (V-RL) ausgesprochen hatte, die geeignet wäre, den Schutz europäischer Vogelarten vor ungewollten, aber in Kauf genommenen Beeinträchtigungen zu mindern, ${ }^{1}$ wurde die Entscheidung des Gerichtshof der Europäischen Union $(\mathrm{EuGH})$ mit Spannung erwartet. Um es vorwegzunehmen: Den Ansatz der Generalanwältin griff der Gerichtshof nicht auf, sondern beantwortet die ihm durch ein schwedisches Gericht unterbreiteten Fragen zur Auslegung des Art. $5 \mathrm{~V}-\mathrm{RL}$ und der Parallelvorschrift des Art. 12 Abs. 1 der FFH-Richtlinie (FFH-RL) in einer Weise ${ }^{2}$ die sich auch für das Verständnis und die Anwendung des bundesdeutschen Artenschutzrechts (IS $44 \mathrm{ff}$. BNatSchG) als folgenreich erweist.

\section{Gefährdungsunabhängiger Schutz der Vogelarten}

Im Ausgangsverfahren wandte sich der Verein „Schützt den Wald" gegen die Abholzung eines Waldgebietes in Schweden, die zur Folge hätte, dass Exemplare geschützter Vogelund Amphibienarten getötet oder gestört und ihre Eier der Vernichtung anheimgegeben würden. Mit Blick auf Art. 5 V-RL warf das schwedische Gericht die Frage auf, ob diese Bestimmung lediglich Arten erfasst, die in Anhang I V-RL

apl. Prof. Dr. Martin Gellermann, Rechtsanwalt, Westerkappeln, Deutschland

Ass. jur. Jochen Schumacher,

Institut für Naturschutz und Naturschutzrecht Tübingen,

Tübingen, Deutschland aufgeführt, auf irgendeiner Ebene bedroht oder deren Populationen auf lange Sicht rückläufig sind.

Vor dem Hintergrund des schon in den Begründungserwägungen der im Jahre 1979 erlassenen VogelschutzRichtlinie thematisierten, aber bis heute nicht gestoppten Rückgangs der Bestände europäischer Vogelarten erinnert der EuGH daran, dass die Umweltpolitik der Union auf ein hohes Schutzniveau abzielt (Art. 191 Abs. 2 AEUV). Zugleich stellen die Luxemburger Richter unter Verweis auf den Wortlaut des Art. 5 V-RL, den systematischen Zusammenhang sowie den Regelungszweck klar, dass die dort geregelten Verbote sämtlichen europäischen Vögeln unabhängig davon zu Gute kommen, ob sie bedroht oder gefährdet sind oder ihre Populationen einen negativen Trend aufweisen. Eine Verwaltungspraxis, die (noch) ungefährdeten Vogelarten unter Hinweis darauf den Schutz der zur Umsetzung des Art. $5 \mathrm{~V}$-RL bestimmten nationalen Rechtsvorschriften versagt, dass es sich um verbreitete und anpassungsfähige Arten mit stabilen Populationen handelt, ist mit dem europäischen Artenschutzrecht nicht vereinbar. Das fügt sich in die bisherige Rechtsprechung ein, hat der Gerichtshof doch schon im Santoña-Urteil betont, dass die richtliniengestützten Verpflichtungen zum Schutz der Vogelarten schon bestehen, bevor eine Abnahme der Zahl der Vögel festgestellt wird oder sich die Gefahr des Aussterbens einer geschützten Vogelart konkretisiert hat. ${ }^{3}$ In

1) GA Kokott, SchlA v. 10.9.2020, C-473/19 und C-474/19, Skydda Skogen, ECLI:EU:C:2020:699; hierzu Gellermann/Schumacher, Absicht ist nicht gleich Absicht?, NuR 2020, $841 \mathrm{ff}$.

2) EuGH, Urt. v. 4.3.2021 - C- 473/19 und C-474/19, Skydda Skogen ECLI:EU:C:2021:166.

3) EuGH, Urt. v. 2.8.1993 - C-355/90, Kommission/Spanien, Slg. 1993, I-4221 Rdnr. 15; ferner Urt. v. 14.1.2016 - C-141/14, NuR 2016, 112 Rdnr. 76; Urt. v. 24.11.2016 - C-461/14, NuR 2017, 36 , Rdnr. 83; Urt. v. 17. 4. 2018 - C-441/17, NuR 2018, 327, Rdnr. 262 